\title{
Disposition Event Start Day
}

National Cancer Institute

\section{Source}

National Cancer Institute. Disposition Event Start Day. NCI Thesaurus. Code C83252.

The day of the week a disposition event began. 